\title{
Experimental Study on Dynamic Properties of High Strength Fiber Clusters
}

\author{
Chenguang Huang ${ }^{1, a}$, Siying Chen ${ }^{1,2, b}$, Zhuping Duan ${ }^{1, c}$ \\ ${ }^{1}$ The State Key Laboratory of Nonlinear Mechanics, Institute of Mechanics, Chinese \\ Academy of Sciences, 100080, Beijing, China \\ ${ }^{2}$ Photoelectricity Department, Beijing Institute of Technology, 100081, Beijing, China \\ a huangcg@imech.ac.cn, ${ }^{\mathrm{b}}$ csy@bit.edu.cn, ${ }^{\mathrm{c}}$ zpduan@Inm.imech.ac.cn
}

\section{Keywords: High strain rate, High strength fiber, Microstructure}

\begin{abstract}
In this paper, the dynamic behaviors of several kinds of high strength fibers, including Kevlar, UHMPE, glass fibers, carbon fibers etc., are investigated experimentally, with a Split Hopkinson Tension Bar (SHTB). The effect of strain rate on the modulus, strength, failure strain and failure characteristics of fibers, under impact loading, is analyzed with the relative stress vs. strain curves. At the same time, the mechanism about the rate dependence of mechanical behaviors of various fibers is discussed based on the understanding on the microstructures and deformation models of materials. Some comments are also presented on the decentralization of experimental results, and a new method called traveling wave method is presented to increase the experimental accuracy. Research results obtained in this paper will benefit to understand the energy absorption and to build up the constitutive law of protective materials reinforced by high strength fibers.
\end{abstract}

\section{Introduction}

Composites have played a very important role in the aerospace vehicle and other engineering as a structural material. Specially, the fiber reinforced polymer has many successful applications. Recently, the research on high strength fibers attracts many scientist's interests, because laminated composites, which are reinforced by Kevlar, ultra high modulus polyethylene (UHMPE), glass fiber, carbon fibers etc., are considered as effective energy-absorbing and guard materials, and can be used in the shield of structures and body armor [1,2].

In general, there are two kinds of high strength fiber: inorganic fiber and organic fiber. The former is represented by the glass fiber and carbon fiber, in the later, Kevlar, UHMPE etc. are the most common used in engineering.

In order to optimize the guard structure made of laminates reinforced by high strength fibers, the properties of composites, such as strength, stiffness, toughness, failure strain and de-bonding criterion, etc. must be known. However, the volume content of fibers in this kind of laminate, is up to about $80 \%$, the understanding about mechanical properties of high strength fibers under different loading conditions is necessary.

The quasi-static properties of various fibers, and their microscopic deformation characteristics, have been studied for decades of years, and can be found in some handbooks. Now, some scientists and engineers pay more attentions in the dynamic properties of the high strength fibers under impact loading.

Ward, Brown [3,4] investigated experimentally the impacting tensile behaviors of Nylon 66, Kevlar 29, Spectra and PBT, and found that strength, modulus and elongation of the above fibers decrease greatly, compared to that of quasi-static. In Ward's experiments [4], the explosive loading was adapted to make the deformation rate of fibers up to $305 \mathrm{~m} / \mathrm{s}$. Xiong [5] investigated the impacting properties of PVA 7901 and Twaron fibers at the 